BMJ Open

Diabetes

Research

\& Care

\section{Sex differences in circulating leptin as a marker of adiposity in obese or overweight adolescents with type 1 diabetes}

To cite: Redondo MJ, Siller AF, Gu X, et al. Sex differences in circulating leptin as a marker of adiposity in obese or overweight adolescents with type 1 diabetes. BMJ Open Diab Res Care 2020;8:e001683. doi:10.1136/ bmjdrc-2020-001683

Part of the contents of this manuscript were presented at the American Diabetes Association Scientific Meetings in June 2019

Received 12 June 2020 Revised 4 September 2020 Accepted 10 September 2020

Check for updates

(C) Author(s) (or their employer(s)) 2020. Re-use permitted under CC BY-NC. No commercial re-use. See rights and permissions. Published by BMJ.

For numbered affiliations see end of article.

Correspondence to Dr Maria J Redondo; redondo@bcm.edu

\section{ABSTRACT}

Introduction We aimed to test whether the serum adipokines leptin and adiponectin are more strongly associated with body fat percentage (BF\%) than body mass index (BMI) in adolescents with type 1 diabetes (T1D) and overweight/obesity.

Research design and methods We studied all participants in the T1D Exchange Metformin Study $(n=122$, median age 12.9 years, range $12-19.5 ; 32 \%$ males; $77 \%$ non-Hispanic whites, $100 \%$ overweight or obesity; median diabetes duration 6.7 years, range 1.4-15) with a baseline serum sample where we measured leptin and adiponectin concentrations. Anthropometric, clinical, laboratory and dual-energy X-ray absorptiometry (DEXA) scan measurements were analyzed. We compared correlation coefficients between variables of interest.

Results $\mathrm{BF} \%$ by DEXA was significantly correlated with BMI Z-score $(r=0.38, p<0.0001)$, BMI per cent of the 95th percentile (BMI\%95) $(r=0.45, p<0.0001)$, waist circumference $(r=0.46, p<0.0001)$, leptin $(r=0.58$, $p<0.00001)$ and leptin/adiponectin ratio $(r=0.36$, $p<0.0001$ ), while it was not significantly correlated with absolute body weight, adiponectin or insulin dose (total or basal). BF\% was significantly more strongly correlated with leptin than with BMI Z-score in the overall group $(p=0.022)$. However, there were sex-based differences. Among the significant correlations in the overall group, $\mathrm{BF} \%$ was most strongly associated with leptin $(r=0.75)$ in boys $(\mathrm{n}=39)$ but with waist circumference $(\mathrm{r}=0.58)$ in girls $(n=83)$ (all $p<0.0001)$.

Conclusions Serum leptin could be used as a surrogate convenient marker of adiposity in overweight/obese adolescent boys with $\mathrm{T1D}$, equivalent to $\mathrm{BMI} \mathrm{Z}$-score or BMI\%95. In girls, waist circumference was the best performing marker overall, and was also strongly correlated with \%BF in boys.

\section{INTRODUCTION}

Obesity is a comorbidity in $30 \%-40 \%$ of US youth with type 1 diabetes (T1D). ${ }^{1}$ Obesity is associated with higher prevalence of cardiovascular risk markers among children with T1D possibly through adiposity-induced chronic inflammation. ${ }^{2}$ Visceral fat accumulation specifically has been linked to an

\section{Significance of this study}

What is already known about this subject?

- Obesity is an important comorbidity in type 1 diabetes (T1D) but its assessment is challenging.

- Measures of body composition are not widely available in clinical practice or large research studies.

- Anthropometric measures are imperfect correlates and often are not available.

- Adipokines are proteins secreted by the adipose tissue that regulate obesity-induced inflammation and can be measured in serum or plasma.

- Whether serum adipokines could be used as convenient, surrogate markers of adiposity in obese/overweight adolescents with T1D is not known.

What are the new findings?

- Body fat percentage (BF\%) was significantly more strongly correlated with leptin than with body mass index Z-score in the overall group.

- Leptin was the marker most strongly associated with $\mathrm{BF} \%$ in boys.

- Waist circumference was the marker, among those that were statistically correlated in the overall group, most strongly associated with $\mathrm{BF} \%$ in girls.

How might these results change the focus of research or clinical practice?

- Serum leptin could be used as a surrogate convenient marker of adiposity in overweight/obese adolescent boys with T1D.

- Waist circumference seems the best marker for \%BF in girls and was also strongly correlated with \%BF in boys.

- The sex bias in the relationship between adipokines and $\mathrm{BF} \%$ warrants further studies.

increased risk of diabetes and cardiovascular disease. ${ }^{3}$ However, body composition measurements are difficult to obtain in both community research and clinical settings. While obesity is defined on the basis of body mass index (BMI) values, $\mathrm{BMI}$ is an imperfect measure of adiposity. ${ }^{4}$ Furthermore, obtaining 
anthropometric values necessary for BMI calculations is often challenging in the research setting.

Adipokines are proteins secreted by the adipose tissue that regulate obesity-induced inflammation and can be measured in serum or plasma. Pro-inflammatory adipokines include leptin, while adiponectin has antiinflammatory actions. We previously showed among children with new-onset T1D, those with obesity had significantly higher serum leptin and lower adiponectin compared with the children who were lean. ${ }^{5}$ However, whether adipokines can be used as a surrogate marker of adiposity in adolescents with T1D has not been evaluated. Therefore, we aimed to test the hypothesis that leptin and adiponectin are more strongly associated with body fat percentage (BF\%) than BMI in adolescents with T1D and overweight/obesity. If our hypothesis is true, serum adipokine levels could be used instead of BMI as a more convenient marker of obesity in both clinical and research settings, perhaps both as a diagnostic tool and a therapeutic target to improve clinical outcomes in individuals with T1D and obesity.

\section{METHODS}

Participants

The T1D Exchange Metformin Study ${ }^{6}$ assessed the efficacy and safety of metformin as an adjunct to insulin in treating obese/overweight adolescents with T1D. T1D was defined based on continuous use of exogenous insulin with either an age of diagnosis $<10$ years of age or positive islet cell antibodies. A total of 140 youth aged 12 to $<20$ years, with T1D for at least 1 year and BMI $\geq 85$ th percentile participated in this randomized controlled trial. They were recruited across 26 clinical sites of the T1D Exchange Clinic Network. In this analysis, we included all the participants in both arms, metformin and control, who had a baseline serum sample $(n=122)$.

\section{Procedures}

Participants in the T1D Exchange Metformin Study underwent extensive characterization including anthropometric and clinical measurements (height, weight, waist circumference, blood pressure), laboratory measurements (eg, lipid levels, liver enzymes, C-peptide and a panel of adipokines, cytokines and other inflammatory markers) and imaging test (dual-energy X-ray absorptiometry (DEXA) scan), as previously published. ${ }^{6}$ Of note, the adipokine panel did not include leptin. All registry study materials have been reviewed and approved by Western IRB (Olympia, Washington, USA).

\section{Laboratory tests}

Serum from all available subjects who provided biological samples at baseline (randomization) $(n=122)$ was obtained from the T1D Exchange. The samples were obtained in fasting conditions between October 2013 and February 2014, and stored in frozen at -80C for an average of 5 years until they were assayed. Leptin and adiponectin levels were measured using Milliplex Human adipocyte magnetic bead assay which has an inter-assay and intra-assay coefficient of variation $<10 \%$.

\section{Statistical analysis}

We used descriptive statistics to summarize characteristics in the overall cohort as well as by group. Chi-square tests were used to compare proportions among groups. Continuous measures were compared between groups using t-tests. Correlation coefficients were calculated between variables of interest, and then compared using computer software available from http://quantpsy.org. We used weight, height, age and gender collected from the registry to calculate BMI (weight in $\mathrm{kg} /$ height in $\mathrm{m}^{2}$ ) and the BMI Z-score based on the Center for Disease Control standards (https://www.cdc.gov/growthcharts/ zscore.htm). We also calculated BMI per cent of the 95th percentile (BMI\%95) as the subject's BMI for age and gender divided by the BMI equal to the 95 th percentile for their age and gender ((https://www.cdc.gov/ growthcharts/percentile_data_files.htm) and multiplied by 100 (subject's BMI/BMI at the 95th percentile×100). BMI\%95 has been shown in other studies to better represent weight abnormalities in the severely obese. ${ }^{8}$ All tests were two-tailed. $\mathrm{P}$ values $<0.05$ were considered to be statistically significant.

\section{RESULTS}

We analyzed serum leptin and adiponectin in 122 adolescents (median age 12.9 years, range $12-19.5$; 32\% males; $77 \%$ non-Hispanic whites) with overweight/obesity and T1D who participated in the T1D Exchange Metformin Study. Their median duration of diabetes was 6.7 years (range 1.4-15), mean BMI Z-score was 2.03 (SD 0.79), mean BMI\%95 was $117.6 \%$ (SD 23.2) and mean $\mathrm{BF} \%$ by DEXA scan was $37.4 \%$ (SD 7.83). Median (IQR) serum leptin and adiponectin were, respectively, 8316 (4832$14177) \mathrm{pg} / \mathrm{mL}$ and 18.1 (12.7-30.7) $\mathrm{\mu g} / \mathrm{mL}$. Other baseline characteristics are illustrated in table 1 .

We found that the BF\% by DEXA in these adolescents with overweight/obesity and T1D was significantly correlated with BMI Z-score $(\mathrm{r}=0.38, \mathrm{p}<0.0001)$, BMI\%95 ( $\mathrm{r}=0.45, \quad \mathrm{p}<0.0001)$, waist circumference $(\mathrm{r}=0.46, \quad \mathrm{p}<0.0001)$, leptin $(\mathrm{r}=0.58, \mathrm{p}<0.00001)$ and leptin/adiponectin ratio $(\mathrm{r}=0.36, \mathrm{p}<0.0001)$, while it was not significantly correlated with absolute body weight, adiponectin or insulin dose (total or basal) (table 2).

Next, we compared the strength of the correlation between $\mathrm{BF} \%$ and each of the obesity measures that were significantly correlated with it. We observed that $\mathrm{BF} \%$ was significantly more strongly correlated with leptin than with BMI Z-score $(p=0.022)$ or leptin/adiponectin ratio $(p=0.001)$. On the other hand, the strength of the correlation between $\mathrm{BF} \%$ and leptin was not significantly different than those of BF\% with each of BMI\%95 $(\mathrm{p}=0.11)$ or waist circumference $(\mathrm{p}=0.17)$.

Girls had a higher mean BF\% (40.3\%, SD 5.41) compared with boys $(31.4 \%$, SD 8.6, $\mathrm{p}<0.00001)$. We did 


\begin{tabular}{lc|}
\hline \begin{tabular}{l} 
Table 1 \\
\hline $\begin{array}{l}\text { Age at T1D diagnosis (median, range) } \\
\text { (years) }\end{array}$
\end{tabular} & $8(1-15)$ \\
\hline $\begin{array}{l}\text { Age at randomization (median, range) } \\
\text { (years) }\end{array}$ & $14.9(12-19.5)$ \\
\hline Male (\%) & 32 \\
\hline Race/Ethnicity (\%) & \\
\hline Non-Hispanic white & 77.1 \\
\hline Hispanic & 12.3 \\
\hline African-American & 6.6 \\
\hline Other & 4.1 \\
\hline BMI Z-score & $2.03(0.79)$ \\
\hline BMI\%95 & $117.6(23.2)$ \\
\hline Waist circumference (cm) & $91.0(\mathrm{SD} 9.7)$ \\
\hline Weight (kg) & $76.4(10.5)$ \\
\hline Percentage body fat (DEXA) & $37.43(7.8)$ \\
\hline DEXA total mass (kg) & $75(10.4)$ \\
\hline DEXA fat mass (g) & $27727.2(7523.5)$ \\
\hline DEXA lean mass (g) & $45179.9(7957.8)$ \\
\hline Systolic blood pressure (mm Hg) & $116.2(9.5)$ \\
\hline Diastolic blood pressure (mm Hg) & $68.0(7.0)$ \\
\hline Tanner stage for pubic hair (\%) & 1.6 \\
\hline 1 & 2.5 \\
\hline 2 & 9.0 \\
\hline 3 & 16.4 \\
\hline 5 & 70.5 \\
\hline
\end{tabular}

Tanner stage for breast development (females only) (\%)

\begin{tabular}{lc}
\hline 1 & 1.6 \\
\hline 2 & 0.8 \\
\hline 3 & 8.2 \\
4 & 13.1 \\
\hline 5 & 76.2 \\
\hline Total insulin dose (units/kg/day) & $1.09(0.21)$ \\
\hline Basal insulin dose (units/kg/day) & $0.52(0.12)$ \\
Leptin (pg/mL) (median, IQR) & $8316(4832-14177)$ \\
\hline Adiponectin ( $\mathrm{\mu g} / \mathrm{mL})$ (median, IQR) & $18.1(12.7-30.7)$ \\
\hline Leptin/Adiponectin ratio (median, IQR) & $402(188.0-871.2)$ \\
\hline
\end{tabular}

Measures are mean (SD) unless otherwise indicated.

$\mathrm{BMI}$, body mass index; DEXA, dual-energy X-ray

absorptiometry; T1D, type 1 diabetes.

not find any significant association between $\mathrm{BF} \%$ and age $(p=0.88)$ or race/ethnicity $(p=0.95)$. There were sexbased differences in the relationships between $\mathrm{BF} \%$ and each of the remaining obesity measures (table 3 ).

In boys $(n=39)$, the correlation coefficient between $\mathrm{BF} \%$ and leptin $(\mathrm{r}=0.75)$ tended to be stronger than that between BF\% and BMI-Z score ( $r=0.55 ; \mathrm{p}=0.071$ for the comparison between the two correlation coefficients)
Table 2 Correlation between percentage of body fat by DEXA and markers of adiposity in overweight and obese adolescents with type 1 diabetes

\begin{tabular}{lll}
\hline & $\begin{array}{l}\text { Correlation } \\
\text { coefficient (r) }\end{array}$ & P value \\
\hline Weight & - & 0.12 \\
Waist circumference & 0.46 & 0.00001 \\
BMI Z-score & 0.38 & 0.00001 \\
BMI\%95 & 0.45 & 0.00001 \\
Leptin & 0.58 & 0.00001 \\
Adiponectin & - & 0.65 \\
\hline Leptin/Adiponectin ratio & 0.36 & 0.00001 \\
\hline Total insulin dose (units/kg/day) & - & 0.76 \\
Basal insulin dose (units/kg/day) & - & 0.99 \\
\hline
\end{tabular}

Correlation coefficients are provided only for statistically significant relationships.

$\mathrm{BMI}$, body mass index; DEXA, dual-energy X-ray absorptiometry.

(figure 1, panels A and B). Similarly, the correlation between $\mathrm{BF} \%$ and leptin ( $\mathrm{r}=0.75)$ tended to be stronger than $\mathrm{BF} \%$ and $\mathrm{BMI} \% 95$ ( $\mathrm{r}=0.57$; $\mathrm{p}=0.063$ for the comparison between correlation coefficients). The correlation between $\mathrm{BF} \%$ and leptin ( $\mathrm{r}=0.75$ ) was statistically stronger than those between $\mathrm{BF} \%$ and waist circumference $(r=0.52 ; p=0.027)$ and ratio of leptin/adiponectin $(\mathrm{r}=0.32 ; \mathrm{p}<0.0001)$. In girls $(\mathrm{n}=83)$, the strength of the correlations between $\mathrm{BF} \%$ and each of weight $(\mathrm{r}=0.60)$ and waist circumference $(\mathrm{r}=0.58)$ were not statistically different $(\mathrm{p}=0.77)$, but weight was not statistically correlated with $\mathrm{BF} \%$ in the overall group (table 2). The correlation between $\mathrm{BF} \%$ and waist circumference

Table 3 Correlations between body fat percentage (BF\%) by DEXA and markers of adiposity in overweight and obese by sex

\begin{tabular}{lllllll}
\hline & \multicolumn{3}{c}{ Males } & & \multicolumn{2}{c}{ Females } \\
\cline { 2 - 3 } \cline { 5 - 6 } & $\mathbf{r}$ & P value & & r & P value \\
\hline Weight & - & 0.80 & & 0.60 & 0.0001 \\
Waist circumference & 0.52 & 0.0006 & & 0.58 & 0.00001 \\
BMI Z-score & 0.55 & 0.0003 & & 0.30 & 0.006 \\
BMI\%95 & 0.57 & 0.0002 & & 0.43 & 0.0001 \\
Leptin & 0.75 & 0.00001 & & 0.32 & 0.0032 \\
Adiponectin & - & 0.19 & & 0.63 \\
$\begin{array}{l}\text { Leptin/Adiponectin } \\
\text { ratio }\end{array}$ & 0.32 & 0.0002 & & - & 0.11 \\
$\begin{array}{l}\text { Total insulin dose } \\
\text { (units/kg/day) }\end{array}$ & - & 0.71 & - & 0.99 \\
$\begin{array}{l}\text { Basal insulin dose } \\
\text { (units/kg/day) }\end{array}$ & - & 0.67 & - & 0.21 \\
\hline
\end{tabular}

Correlation coefficients are provided only for statistically significant relationships.

BMI, body mass index; DEXA, dual-energy X-ray absorptiometry. 

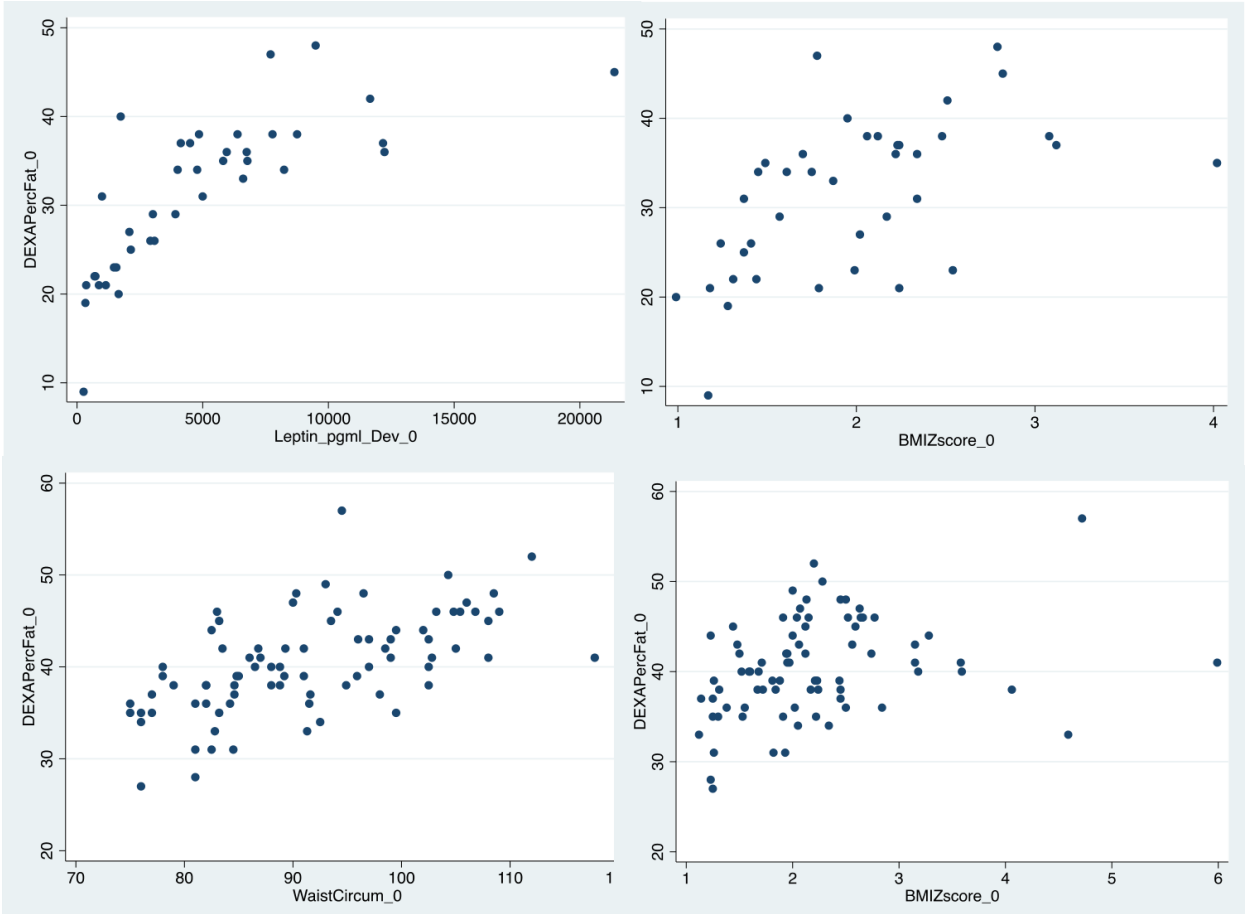

Figure 1 Body fat percentage (BF\%) is more strongly correlated with serum leptin ( $r=0.75, p=0.00001$, panel $A)$ than with body mass index (BMI) Z-score $(r=0.55, p=0.0003$, panel $B)$ in boys $(n=39, p=0.07)$, while it is more strongly correlated with waist circumference $(r=0.58, p=0.0001$, panel $C)$ than with BMI Z-score $(r=0.30, p=0.006$, panel $D)$ in girls $(n=83, p=0.01)$. DEXA, dual-energy X-ray absorptiometry.

$(\mathrm{r}=0.58)$ was statistically stronger than those of $\mathrm{BF} \%$ and leptin $(\mathrm{r}=0.32, \mathrm{p}=0.025)$ and $\mathrm{BF} \%$ and BMI Z-score $(\mathrm{r}=0.30, \mathrm{p}=0.01)$ (figure 1 , panels $\mathrm{C}$ and $\mathrm{D})$, but not statistically stronger than that of $\mathrm{BF} \%$ with $\mathrm{BMI} \% 95(\mathrm{r}=0.43$; $\mathrm{p}=0.13$ ).

\section{DISCUSSION}

The goal of this project was to test whether a serum marker (ie, leptin, adiponectin) could be used as a measure of $\mathrm{BF} \%$ instead of BMI Z-score, percentile or waist circumference. We observed that leptin and $\mathrm{BF} \%$ are statistically and strongly correlated and thus, leptin could be an adequate marker of $\mathrm{BF} \%$ in overweight/ obese adolescent boys with T1D. Furthermore, we observed a trend for $\mathrm{BF} \%$ being more strongly correlated with leptin than with BMI Z-score or BMI\%95; therefore, serum leptin could be used as a convenient marker of adiposity in overweight/obese adolescent boys with T1D, certainly not worse than BMI Z-score or BMI\%95, and possibly more accurate reflection of $\mathrm{BF} \%$ (figure 1 ). In boys, serum leptin was more strongly correlated with $\mathrm{BF} \%$ than waist circumference or the leptin/adiponectin ratio. Height and weight are in dynamic flux during the growth spurt years of adolescence and BMI calculation uses total weight (bone, muscle, fat, connective tissue, etc). Individual differences in growth velocity, bone lengthening, muscle mass, etc, during the growing phase could affect the correlation between BMI and body fat. In contrast, leptin is secreted by fat cells, which can explain why its levels correlate better with body fat than BMI.
Many research studies performed in community settings do not obtain anthropometric data and thus secondary analyses of their outcomes with measures of obesity are not possible. On the other hand, serum is often available from these studies that thus allow researchers to measure metabolites such as adipokines. Our study shows that serum leptin can be used as a marker of adiposity in overweight/obese adolescent boys with T1D, which will facilitate these studies. Further research on the relationship of leptin with outcomes of interest such as insulin resistance and cardiovascular disease is needed to understand if serum leptin could be advantageous over anthropometric markers of adiposity for diagnosis and as a therapeutic target in the clinical setting, where height and weight are usually available.

In girls, out of the measures that were statistically correlated with $\mathrm{BF} \%$ in the overall group, waist circumference was the best performing, and was more strongly correlated with $\mathrm{BF} \%$ than BMI Z-score (figure 1, panels $\mathrm{C}$ and $\mathrm{D}$ ) and leptin. While in the overall cohort of boys and girls leptin was strongly correlated with adiposity, and the relationship was statistically significant in each of the male and female groups, the strength was relatively weak in girls. Therefore, serum leptin, although convenient, may not reflect adiposity better than anthropometric measures in studies that include adolescent girls. Since it is often necessary to evaluate the same variable in males and females, waist circumference was the measure that performed best separately in boys and girls, and, after leptin, also in the combined group. 
There are several studies showing that the leptin/ adiponectin ratio is an emerging biomarker to measure insulin resistance. ${ }^{9}$ In our study, leptin/adiponectin ratio was statistically correlated with $\mathrm{BF} \%$ in the overall cohort but the stratified analysis indicated that this held true in boys but not in girls.

The reason for the different performance of leptin as a marker of adiposity between boys and girls is unclear. A previous study in children with obesity showed higher levels of leptin in otherwise healthy children with obesity compared with lean. ${ }^{10}$ In our data, although girls had higher percentage of body fat and narrower range (mean $40.3 \%$, SD 5.4) than boys (mean $31.4 \%$, SD 8.6), the correlation between leptin and adiposity was not strong. A potential explanation is the narrower range of $\mathrm{BF} \%$ in girls than boys in our data. Supporting this hypothesis, in a study of children with obesity with a narrow range of BMI distribution, leptin had a slightly less strong correlation to $\mathrm{BF} \%$ compared with BMI or waist circumference despite being predominantly male. ${ }^{10}$ However, there were additional differences between the studies, such as the populations (overweight and obese children with T1D vs healthy children with obesity) and methodology to measure body fat (DEXA vs bioimpedance) that could also explain the different results. Clearly, more research is needed to understand this sex-based heterogeneity. In addition, our observation of dissimilarities between boys and girls in the relationship between markers of adiposity and $\mathrm{BF} \%$ (table 3) support the need for studies in this field to address potential sex differences.

A unique characteristic of our subject population was the administration of exogenous insulin. While insulin is crucial for glucose control, it is also important for adipogenesis. In addition, insulin is a leptin secretagogue. ${ }^{11} \mathrm{~A}$ previous study found that changing insulin delivery from multiple daily injections to a continuous subcutaneous system may influence leptin levels in women with T1D. ${ }^{12}$ While we did not see any association between adipokines in our adolescent subjects with T1D and their total daily insulin dose, it is possible that residual endogenous insulin secretion and exogenous insulin administration had influence on the leptin levels observed in the study participants.

We did not see any association of race/ethnicity on leptin levels. However, leptin levels have been previously shown to be higher in African-American and nonHispanic whites compared with Hispanic subjects with T1D. ${ }^{13}$ Thus, it is possible that effects of race/ethnicity were masked by the more homogenous population in these data since our sample included a predominantly white population $(75 \%){ }^{6}$

There are some limitations to this study. Body fat distribution is important for metabolic risk, with visceral fat contributing more to metabolic derangements than subcutaneous fat. ${ }^{14}$ This dataset did not include differences in visceral versus subcutaneous fat distribution, so these correlations could not be performed. There was marked heterogeneity in serum leptin levels among individuals of similar \%BF, although less than for other markers of adiposity, as seen in figure 1 . This wide range for leptin is consistent with prior reports, ${ }^{15}$ and could be due, in part, to intrinsic differences in insulin sensitivity. ${ }^{16}$ The relatively modest sample sizes studied for boys and girls could have affected the findings regarding strengths of correlations.

In conclusion, we studied serum adipokine levels in overweight/obese adolescents with T1D who participated in the T1D Exchange Metformin Study. Our findings suggest that serum leptin could be used as an accurate marker of adiposity in boys. In girls, waist circumference was the best performing marker and was also strongly correlated with $\% \mathrm{BF}$ in boys.

\section{Author affiliations}

${ }^{1}$ Texas Children's Hospital, Diabetes and Endocrinology Section, Baylor College of Medicine, Houston, Texas, USA

${ }^{2}$ Epidemiology and Population Science Section, Department of Medicine, Baylor College of Medicine, Houston, Texas, USA

${ }^{3}$ Epidemiology and Population Health, Stanford Cancer Institute, Stanford, California, USA

${ }^{4}$ Department of Pathology, Texas Children's Hospital, Baylor College of Medicine, Houston, Texas, USA

${ }^{5}$ Children's Nutrition Research Center, Department of Pediatrics, Baylor College of Medicine, Houston, Texas, USA

Acknowledgements The authors are grateful to the T1D Exchange and the patients who provided their time and effort in contributing critical information. Without their assistance, this research would not have been possible. The authors would like to thank Kara Page for her assistance with coordinating study sample shipments and data transfer.

Contributors MJR designed the study, obtained approval from the T1D Exchange, contributed to data analysis and interpretation and wrote the first draft of the manuscript. AFS, MT and MB contributed to data interpretation and manuscript revisions. XG conducted statistical analyses and contributed to manuscript revisions. SD supervised laboratory methods and contributed to data interpretation and manuscript revisions. SS critically contributed to conceptual data interpretation, manuscript writing and revisions. All authors have approved the version of the manuscript to be published.

Funding This work was supported by the American Diabetes Association (1-17JDF-037) to SS and the US Department of Agriculture, Agriculture Research Service (cooperative agreement no. 58-6250-6-001) to SS.

Competing interests SS receives support from Rhythm Pharmaceuticals for scientific advisory boards and for Speakers Bureau engagements.

Patient consent for publication Not required.

Ethics approval The Metformin Therapy for Overweight Adolescents with Type 1 Diabetes (NCT01881828) was sponsored by the Jaeb Center for Health Research with collaboration from the Juvenile Diabetes Research Foundation. The study was approved by the Jaeb Center for Health Research IRB (registration number: IRB00000766), and by local institutional review boards at each participating institution. All participants and/or their guardians provided written informed consent.

Provenance and peer review Not commissioned; externally peer reviewed.

Data availability statement Data are available in a public, open access repository. T1D Exchange Metformin Study data are publicly available. Serum leptin and adiponectin data are available on reasonable request.

Open access This is an open access article distributed in accordance with the Creative Commons Attribution Non Commercial (CC BY-NC 4.0) license, which permits others to distribute, remix, adapt, build upon this work non-commercially, and license their derivative works on different terms, provided the original work is properly cited, appropriate credit is given, any changes made indicated, and the use is non-commercial. See: http://creativecommons.org/licenses/by-nc/4.0/.

\section{ORCID iDs}

Maria J Redondo http://orcid.org/0000-0001-5871-4645

Mustafa Tosur http://orcid.org/0000-0002-2111-271X 


\section{REFERENCES}

1 Minges KE, Whittemore R, Weinzimer SA, et al. Correlates of overweight and obesity in 5529 adolescents with type 1 diabetes: the T1D exchange clinic registry. Diabetes Res Clin Pract 2017;126:68-78.

2 Redondo MJ, Foster NC, Libman IM, et al. Prevalence of cardiovascular risk factors in youth with type 1 diabetes and elevated body mass index. Acta Diabetol 2016;53:271-7.

3 Jensen MD. Role of body fat distribution and the metabolic complications of obesity. J Clin Endocrinol Metab 2008;93:s57-63.

4 Shah NR, Braverman ER. Measuring adiposity in patients: the utility of body mass index (BMI), percent body fat, and leptin. PLoS One 2012;7:e33308.

5 Redondo MJ, Rodriguez LM, Haymond MW, et al. Serum adiposity-induced biomarkers in obese and lean children with recently diagnosed autoimmune type 1 diabetes. Pediatr Diabetes 2014;15:543-9.

6 Libman IM, Miller KM, DiMeglio LA, et al. Effect of metformin added to insulin on glycemic control among overweight/obese adolescents with type 1 diabetes: a randomized clinical trial. JAMA 2015;314:2241-50.

7 Lee IA, Preacher KJ. Calculation for the test of the difference between two dependent correlations with one variable in common, 2013. Available: http://quantpsy.org

8 Gulati AK, Kaplan DW, Daniels SR. Clinical tracking of severely obese children: a new growth chart. Pediatrics 2012;130:1136-40.
9 Frühbeck G, Catalán V, Rodríguez A, et al. Adiponectin-leptin ratio: a promising index to estimate adipose tissue dysfunction. relation with obesity-associated cardiometabolic risk. Adipocyte 2018;7:57-62.

10 Pires A, Martins P, Pereira AM, et al. Insulin resistance, dyslipidemia and cardiovascular changes in a group of obese children. Arq Bras Cardiol 2015;104:266-73.

11 Askari H, Liu J, Dagogo-Jack S. Hormonal regulation of human leptin in vivo: effects of hydrocortisone and insulin. Int $J$ Obes Relat Metab Disord 2000;24:1254-9.

12 Prídavková D, Samoš M, Kazimierová I, et al. Insulin pump therapy - influence on body fat redistribution, skeletal muscle mass and Ghrelin, Leptin changes in T1D patients. Obes Facts 2018;11:454-64.

13 Snell-Bergeon JK, West NA, Mayer-Davis EJ, et al. Inflammatory markers are increased in youth with type 1 diabetes: the search case-control study. J Clin Endocrinol Metab 2010;95:2868-76.

14 Karlsson T, Rask-Andersen M, Pan G, et al. Contribution of genetics to visceral adiposity and its relation to cardiovascular and metabolic disease. Nat Med 2019;25:1390-5.

15 Considine RV, Sinha MK, Heiman ML, et al. Serum immunoreactiveleptin concentrations in normal-weight and obese humans. $N$ Engl $J$ Med 1996;334:292-5.

16 Askari $\mathrm{H}$, Tykodi G, Liu J, et al. Fasting plasma leptin level is a surrogate measure of insulin sensitivity. J Clin Endocrinol Metab 2010;95:3836-43.

17 American Diabetes Association. Economic costs of diabetes in the U.S. in 2012. Diabetes Care 2013;36:1033-46. 